\title{
COMBINED EFFECT OF FREE AND FORCED CONVECTION ON MHD FLOW IN A ROTATING POROUS CHANNEL
}

\author{
D.R.V. PRASADA RAO and D.V. KRISHNA \\ Department of Mathematics \\ Post Graduate Centre, Anantapur \\ Andhra Pradesh, India \\ and

\section{LOKENATH DEBNATH*} \\ Mathematical Institute \\ University of Oxford \\ Oxford, England \\ (Received September 2, 1979 and in revised form July 2, 1980)
}

ABSTRACT. This paper gives a steady linear theory of the combined effect of the free and forced convection in rotating hydromagnetic viscous fluid flows in a porous channel under the action of a uniform magnetic field. The flow is governed by the Grashof number G, the Hartmann number $H$, the Ekman number $E$, and the suction Reynolds number $S$. The solutions for the velocity field, temperature distribution, magnetic field, mass rate of flow and the shear stresses on the channel boundaries are obtained using a perturbation method with the small parameter S. The nature of the associated boundary layers is investigated for various values of the governing flow parameters. The velocity, the temperature, and the shear stresses are discussed numerically by drawing profiles with reference to the variations in the flow parameters.

KEY WORDS AND PHRASES. Free and Forced Convection in a rotating fluid and Magnetohydrodynamic Boundary Layers.

1980 MATHEMATICS SUBJECT CLASSIFICATION CODES. $76 \mathrm{R05}, 76 R 10,76(1) 5$.

1. INTRODUCTION.

In the last several years considerable attention has been given to the study 
of the hydrodynamic thermal convection due to its numerous applications in geophysics and astrophysics. Several authors including Gupta [1], Jana [2], Nanda and Mohanty [3], Mishra and Mudali [4], Mohan [5], Soundalgekar [6], Yen [7], and Gill and Casal [8], have investigated the effects of the free and/or forced convection on hydromagnetic fluid flows confined to non-porous boundaries under various geometrical configurations. Some of these authors have also considered the effects of wall conductance on the heat transfer aspect of hydromagnetic channel flows. It has been shown that the wall conductance exerts a destabilizing influence on the flow whereas the magnetic field stabilizes the flow.

In spite of these studies, relatively less attention has been given to the simultaneous effects of the free and forced convection on the hydromagnetic rotating viscous flows confined to porous boundaries. Such work seems to be important and useful partly for gaining basic understanding of such flows, and partly for possible applications to geophysical and astrophysical problems. The present paper deals with a steady linear theory of the combined effect of the free and forced convection in rotating hydromagnetic viscous fluid flows in a porous channel under the action of a uniform magnetic field. The solutions for the velocity field, temperature distribution, magnetic field, mass rate of the flow and the shear stresses on the channel boundaries are investigated using a perturbation method with the small suction Reynolds number $\mathrm{S}$. The structure of the associated boundary layers is examined for various values of the governing flow parameters. The velocity, the temperature, and the shear stresses are discussed numerically by drawing profiles with reference to the variations in the flow parameters.

2. MATHEMATICAL FORMULATION OF THE PROBLEM.

We consider an incompressible electrically conducting viscous steady fluid flows in a channel bounded by two porous non-conducting parallel plates at $z= \pm L$. Both the fluid and the boundary plates are in a state of solid body rotation with a uniform angular velocity $\Omega$ about the z-axis normal to the plates. We take a Cartesian coordinate system $0 x y z$ rotating with the angular velocity $\Omega$ with the $x-$ axis taken in the direction of pressure gradient and the z-axis positive upward. 
A uniform magnetic field of strength $B_{0}$ parallel to the z-axis is applied to the fluid system, and the fluid is driven by a constant horizontal pressure gradient. The present analysis is based on the Boussinesq approximation which implies that $\alpha \theta \ll 1$, when the density is given by $\rho=\rho_{0}(1-\alpha \theta), \rho_{0}$ is the density at the temperature $T_{0}, \theta$ is the temperature variation from $T_{0}$, and $\alpha$ is the coefficient of thermal expansion. The Boussinesq approximation also implies that the properties of the liquid, $\alpha, v$ the kinematic viscosity and $k$ the thermal diffusivity, are independent of temperature and hence can be taken as constants.

With the Cartesian coordinate system and the Boussinesq approximation, the unsteady motion of a viscous conducting fluid in the presence of a magnetic field $\underline{B}$ in this rotating coordinate system is governed by the Navier-Stokes equations, the continuity equation and the energy equation with usual notations

$$
\begin{gathered}
\frac{\partial \underline{u}}{\partial t}+(\underline{u} \cdot \nabla) \underline{u}+2 \Omega \underline{k} \times \underline{u}=-\nabla P *-\underline{k g}(1-\alpha \theta)+\frac{1}{\rho_{0}} \dot{j} \times \underline{B}+\nu \nabla^{2} \underline{u} \\
\operatorname{div} \underline{u}=0, \\
\frac{\partial \theta}{\partial t}+(\underline{u} \cdot \nabla) \theta=\kappa \nabla^{2} \theta
\end{gathered}
$$

where the velocity $\underline{u}=(u, v, w), P^{*}$ is the pressure including the centrifugal force, $j$ is the current density and $g$ is the acceleration due to gravity. We further assume that $\underline{j}, \underline{B}$ and the electric field $\underline{E}$ satisfy the Maxwe11 equations with usual notations.

The flow is assumed to be fully developed and steady so that all the physical variables except the pressure, depends only on $z$. It then follows from the continuity equation (2.2) that $w=-w_{0}$, a constant. Clearly, $w_{0}>0$ for suction and $\mathrm{w}_{\mathrm{o}}<0$ for injection. Thus the governing flow equations are

$$
\begin{aligned}
w_{0} \frac{\partial u}{\partial z}-2 \Omega v & =-\frac{\partial P *}{\partial x}+v \frac{\partial^{2} u}{\partial z^{2}}+\frac{B_{o}}{\rho_{o} \mu_{e}}\left(\frac{\partial B}{\partial z}\right), \\
w_{0} \frac{\partial v}{\partial z}+2 \Omega u & =v \frac{\partial^{2} v}{\partial z^{2}}+\frac{B_{o}}{\rho_{o}^{\mu} e}\left(\frac{\partial B_{x}}{\partial z}\right), \\
0 & =-\frac{\partial P *}{\partial z}-\frac{1}{\rho_{o}^{\mu} e}\left(B_{y} \frac{\partial B}{\partial z}+B_{x} \frac{\partial B}{\partial z}\right)-g(1-\beta \theta),
\end{aligned}
$$




$$
\begin{aligned}
\left(u \frac{\partial}{\partial x_{0}}+w_{0} \frac{\partial}{\partial z}\right) \theta= & \alpha \frac{\partial^{2} \theta}{\partial z^{2}}+\frac{\nu}{C_{p}}\left[\left(\frac{\partial u}{\partial z}\right)^{2}+\left(\frac{\partial v}{\partial z}\right)^{2}\right]+\frac{1}{\rho_{0} C_{p} \sigma}\left(j_{x}{ }^{2}+j_{y}{ }^{2}\right), \\
& \frac{\partial^{2} B_{x}}{\partial z^{2}}-\frac{w_{o}}{n}\left(\frac{\partial B_{x}}{\partial z}\right)+\frac{B_{0}}{\eta}\left(\frac{\partial u}{\partial z}\right)=0 \\
& \frac{\partial^{2} B_{y}}{\partial z^{2}}-\frac{w_{o}}{n}\left(\frac{\partial B_{y}}{\partial z}\right)+\frac{B_{o}}{n}\left(\frac{\partial}{\partial z}\right)=0
\end{aligned}
$$

where $\left(B_{x}, B_{y}, B_{o}\right)$ are the components of the magnetic field, $\mu_{e}$ is the magnetic permeability, $\sigma$ is the electrical conductivity, $\beta$ is the coefficient of volume expansion, $C_{p}$ is the specific heat and $n=\left(\sigma \mu_{e}\right)^{-1}$ is the magnetic viscosity. The last two terms of the energy equation (2.7) represent the viscous and ohmic dissipation respectively.

Integration of (2.6) gives that

$$
P^{*}=-g z-\frac{1}{2 \rho_{0}^{\mu} e}\left(B_{x}^{2}+B_{y}^{2}+B_{o}^{2}\right)+P_{1} x+\beta g \int \theta d z,
$$

where $P_{1}$ is the uniform pressure gradient with which the fluid is driven along the $\mathrm{x}$-axis.

It is assumed that the porous plates at $z= \pm L$ of thickness $d$ are cooled or heated by a constant temperature gradient $A_{1}$ along the $x$-axis so that the temperature varies linearly along the plates. Eliminating $\mathrm{P*}$ from (2.4) and (2.6), we obtain

$$
\theta=A_{1} x+\theta_{1}(z)
$$

where $\theta_{1}(z)$ is an arbitrary function of $z$.

We next take $T_{0}+A_{1} x+\theta_{10}$ and $T_{0}+A_{1} x+\theta_{11}$ as the temperatures at the lower and upper plates respectively.

It is convenient to write down the non-dimensional form of the basic field equations (2.4) - (2.9) through the non-dimensional variables (starred) defined by the relations

$\left(x^{*}, z^{*}\right)=\frac{1}{L}(x, z),\left(u^{*}, v^{*}\right)=\frac{L}{v}(u, v)$, and $\left(B_{x}^{*}, B_{y}^{*}\right)=\frac{\eta}{v B_{0}}\left(B_{x}, B_{y}\right), \quad(2.12 a b c)$

We next combine the non-dimensional equations (2.4) and (2.5) and use (2.10) 
to obtain the equation (after dropping the asterisks)

$$
\frac{d^{2} F}{d z^{2}}-S \frac{d F}{d z}-2 i\left(\frac{F}{E}\right)+H^{2} \frac{d B}{d z}=G z+R
$$

Similarly, it follows from (2.8) and (2.9) that

$$
\frac{d^{2} B}{d z^{2}}-\operatorname{SPm} \frac{d B}{d z}+\frac{d F}{d z}=0 \text {, }
$$

where $F=u+i v$ is the complex velocity field, $B=B_{x}+i B_{y}$ is the complex magnetic field, $S=\frac{w_{0} L}{v}$ is the cross-flow Reynolds number, $P m=\frac{v}{n}$ is the magnetic Prandt1 number, $H \equiv B_{0} L\left(\frac{\sigma}{v \rho_{0}}\right)^{\frac{1}{2}}$ is the Hartmann number, $G \equiv B g A_{1}\left(\frac{L^{4}}{v^{2}}\right)$ is the Grashof number, $E \equiv \frac{\nu}{\Omega L^{2}}$ is the Ekman number and $R=P_{1}\left(\frac{L^{2}}{\nu^{2}}\right)$.

Substitution of (2.11) into (2.7) yields the energy equation in the nondimensional form

$\frac{1}{P} \frac{d^{2} \tilde{\theta}}{d z^{2}}-S \frac{d \tilde{\theta}}{d z}=G u-E c H^{2}\left\{\left(\frac{d B}{d z}\right)^{2}+\left(\frac{d B}{d z}\right)^{2}\right\}-E c\left\{\left(\frac{d u}{d z}\right)^{2}+\left(\frac{d v}{d z}\right)^{2}\right\}$

where $\mathrm{P}=\frac{\nu}{\mathrm{K}}$ is the non-magnetic Prandtl number, $\mathrm{Ec}=\mathrm{BgL}_{\mathrm{C}} \mathrm{P}$ is the Eckert number and $\tilde{\theta}=\operatorname{BgL}^{3}\left(\theta_{1}-\theta_{10}\right) / \nu^{2}$.

The boundary conditions on the velocity and temperature fields are

$$
\begin{aligned}
& (\mathrm{u}, \mathrm{v})=(0,0) \quad \text { at } \mathrm{z}= \pm 1 \\
& \tilde{\boldsymbol{\theta}}=0 \quad \text { at } z=1 \\
& \tilde{\theta}=g B L^{3}\left(\theta_{11}-\theta_{10}\right) / \nu^{2}=\theta_{0} \text { (say) at } z=-1 \text {, }
\end{aligned}
$$

The boundary conditions on the magnetic field are

$$
\begin{array}{ll}
\frac{\mathrm{dB}}{\mathrm{d} z}+\frac{\mathrm{B}}{\delta}=\frac{\mathrm{B}_{1}}{\delta} & \text { at } z=1, \\
\frac{\mathrm{dB}}{\mathrm{dz}}-\frac{\mathrm{B}}{\delta}=-\frac{\mathrm{B}_{2}}{\delta} & \text { at } z=-1,
\end{array}
$$

where $\delta=\frac{\sigma_{1}}{\sigma} \frac{d}{L}, B_{1}=B_{x 1}+i B_{y 1}, B_{2}=B_{x 2}+i B_{y 2}, \sigma_{1}$ is the electrical conductivity of the plate, $B_{x l}$ and $B_{y l}$ are the values of $B_{x}$ and $B_{y}$ in the vacuum region $z \geqq 1+\frac{d}{L}$, and $B_{x 2}$ and $B_{y 2}$ are the values of $B_{x}$ and $B_{y}$ in the region $z \leqq-1-\frac{d}{L}$. 
The magnetic field within the plates can be obtained using the Maxwell equation $\underline{J}=\sigma_{1} \underline{E}$ and $\operatorname{Cur} 1 \underline{B}=\mu_{\mathrm{J}}$ (since $\underline{\mathrm{u}}=0$ on the plates). If there is a net current through the channel in the y-direction, the induced electric field exists in the y-direction. In view of this, it is observed that the magnetic field components outside the boundary (in the vacuum) exist only in the $x$-direction and vanish in the $y$-direction. The components $B_{x 1}$ and $B_{x 2}$ are fixed by knowing the net current in the y-direction through the channel and its return path.

\section{SOLUTION OF THE PROBLEM.}

We shall adopt a small perturbation method with $S$, the cross-flow Reynolds number as the perturbation parameter, in order to solve the differential equations (2.13) and (2.14). Thus we assume

$$
\begin{aligned}
& F=F_{0}+S F_{1}+0\left(s^{2}\right), \\
& B=B_{3}+S_{4}+0\left(s^{2}\right),
\end{aligned}
$$

Substituting (3.1) - (3.2) into (2.13) and (2.14) and equating the like powers of $\mathrm{S}$, we obtain

$$
\begin{aligned}
& F_{0}^{\prime \prime}-2 i \frac{F_{0}}{E}+H^{2} B_{3}^{\prime}=G z+R, \\
& F_{1}^{\prime \prime}-2 i \frac{F_{1}}{E}+H^{2} B_{4}^{\prime}=F_{0}^{\prime} .
\end{aligned}
$$

and

$$
\begin{aligned}
& \mathrm{B}_{3}^{\prime \prime}+\mathrm{F}_{\mathrm{O}}^{\prime}=0, \\
& \mathrm{~B}_{4}^{\prime \prime}+\mathrm{F}_{1}^{\prime}=\mathrm{Pm}_{\mathrm{O}}^{\prime}
\end{aligned}
$$

where the prime denotes the differentiation with respect to $z$.

The boundary conditions $(2.16)-(2.20)$ reduce to

$$
\left.\left.\begin{array}{l}
\mathrm{F}_{0}=\mathrm{F}_{1}=0 \\
\mathrm{~B}_{3}^{\prime}+\frac{\mathrm{B}_{3}}{\delta}=\frac{\mathrm{B}_{1}}{\delta} \\
\mathrm{B}_{4}^{\prime}+\frac{\mathrm{B}_{4}}{\delta}=0
\end{array}\right\} \begin{array}{l}
\text { on } \mathrm{z}= \pm 1, \\
\mathrm{~B}_{3}^{\prime}-\frac{\mathrm{B}_{3}}{\delta}+\frac{\mathrm{B}_{2}}{\delta}=0 \\
\mathrm{~B}_{4}^{\prime}-\frac{\mathrm{B}_{4}}{\delta}=0
\end{array}\right\} \text { on } \mathrm{z}=1,
$$


The solutions of (3.4) - (3.7) with the above conditions are

$$
\begin{gathered}
F=a_{1}+a_{2} \frac{\cosh \lambda z}{\cosh \lambda}+\frac{a_{3}}{\lambda^{2}}\left\{\frac{\sinh \lambda z}{\sinh \lambda}-z\right\}+\operatorname{Sb}_{7} z, \\
\mathrm{H}^{2} \mathrm{~B}=\mathrm{a}_{9}-\mathrm{a}_{6} \frac{\sinh \lambda z}{\sinh \lambda}-a_{7} \frac{\cosh \lambda z}{\cosh \lambda}+\frac{\mathrm{Sb}_{6} \mathrm{z}^{3}}{3}+\mathrm{a}_{8} \mathrm{Sz}^{2}+\mathrm{a}_{5} z,
\end{gathered}
$$

where $\lambda^{2}=H^{2}+\frac{2 i}{E}, a_{1}=-a_{10}+S\left[2 a_{10} b_{2}-\frac{a_{11}}{a_{10}}-\frac{G H^{2} P m}{2 \lambda^{4}}\right], a_{2}=a_{10}+s\left[\frac{a_{11}}{a_{10}}-2 a_{10} b_{2}\right]$, $a_{3}=G+\operatorname{SPmb}_{3} \lambda^{2}, a_{5}=\frac{2 a_{1}}{E}-\frac{G S b_{2}}{\lambda}+R, a_{6}=\left(a_{2}+2 a_{10} S_{2}\right) \frac{H^{2}}{\lambda}+\frac{a_{10} b_{2} b_{5}}{\lambda}-\frac{G}{\lambda^{2}} \tanh \lambda$, $a_{7}=H^{2}\left[\frac{G}{\lambda^{2}}-\frac{2 S b_{2} G}{\lambda^{3}}+S P m b_{4}\right]+\frac{b_{2} b_{5} G}{\lambda^{3}}-a_{10} \tanh \lambda, a_{8}=\frac{H^{2} G}{2 \lambda^{2}}+\frac{\text { iS Pm } b_{3}}{E}$, $\mathrm{a}_{9}=\frac{\mathrm{GH}^{2}}{\lambda^{3}}(\lambda \delta+\operatorname{coth} \lambda)-\frac{\mathrm{GH}^{2}}{2 \lambda^{2}}(1+2 \delta)+\frac{\mathrm{H}^{2}}{2}\left(\mathrm{~B}_{\mathrm{x} 1}+\mathrm{B}_{\mathrm{x} 2}\right)+\mathrm{H}^{2} \mathrm{~s}(1+\delta) \mathrm{x}$ $(\lambda \delta+\operatorname{coth} \lambda)\left[\frac{\mathrm{Gb}_{2}}{\lambda^{3}}\left(1+\mathrm{b}_{5}\right)-\mathrm{a}_{10} \tanh \lambda+\mathrm{b}_{4} \mathrm{Pm}\right]+\frac{i S \mathrm{Pm}}{\mathrm{E}}(1+2 \mathrm{~S}) \mathrm{b}_{4}$, $a_{10}=\frac{b_{1}(1+\delta)}{\lambda^{2}}+H^{2}(\tanh \lambda-\lambda), a_{11}=\frac{i}{E \lambda^{3}}(1+3 \delta) H^{2} G P m$ $-3(\lambda \delta+\tanh \lambda)\left(\mathrm{b}_{2} \mathrm{~b}_{5} \mathrm{a}_{10}-\frac{G}{\lambda} \operatorname{coth} \lambda\right)-\frac{6 i}{\mathrm{E}}(1+\delta)\left(\frac{\mathrm{H}^{2} G \mathrm{Pm}}{2 \lambda^{3}}-2 \mathrm{~b}_{2} \mathrm{a}_{10}\right)-3(1+\delta) G \mathrm{~b}_{2}$. And the constants $b_{n}$ are given by $b_{1}=R(1+\delta)-\frac{1}{2}\left(B_{x 1}+B_{x 2}\right), b_{2}=\frac{\left(\lambda^{2}+H^{2} P m\right)}{\lambda^{3}}, b_{3}=\frac{1}{\lambda^{2}}\left(R+\frac{21 a 10}{E}\right)$, $b_{4}=\frac{1}{\lambda^{3}}\left(\frac{2 i a_{10}}{E}-R\right), b_{5}=\left(\frac{i}{E}-2 \lambda^{2}\right), b_{6}=\frac{i}{\lambda^{2}} G H^{2} P m$, and $b_{7}=-\frac{i b_{6}}{E}$. If $Q_{x}$ and $Q_{y}$ represent the dimensionless mass flow rates, then

$$
Q_{x}+1 Q_{y}=\frac{2 a_{2}}{\lambda} \tanh \lambda+\frac{2}{3} S b_{7}+2 a_{1} \text {. }
$$

The non-dimensional shear stresses $\tau_{x} \equiv \frac{\partial u}{\partial z}$ and $\tau_{y} \equiv \frac{\partial v}{\partial z}$ are obtained at the upper and lower plates from (3.11), and are given by

$$
\begin{gathered}
\left(\tau_{x}+i \tau_{y}\right)_{z=1}=\lambda a_{2} \tanh \lambda+\frac{a_{3}}{\lambda^{2}}(\lambda \operatorname{coth} \lambda-1)+2 S b_{7}, \\
\left(\tau_{x}+i \tau_{y}\right)_{z=-1}=-a_{2} \lambda \tanh \lambda+\frac{a_{3}}{\lambda^{2}}(\lambda \operatorname{coth} \lambda-1)-2 S b_{7} .
\end{gathered}
$$


To solve the energy equation, it is convenient to write $\Delta=\tilde{\theta}+i \tilde{\phi}$ where $\tilde{\phi}$ is a fictitious function which satisfies the differential equation

$$
\frac{\mathrm{d}^{2} \tilde{\phi}}{\mathrm{dz}} \mathrm{z}^{2}-\mathrm{S} P \cdot \frac{\mathrm{d} \tilde{\phi}}{\mathrm{dz}}=P \quad \text { G v }
$$

with the boundary conditions

$$
\tilde{\phi}(\underline{+1})=0 .
$$

We next combine (2.15) with (3.16) to obtain the equation for $\Delta$

$$
\frac{1}{P} \frac{d^{2} \Delta}{d z^{2}}-S \frac{d \Delta}{d z}+E c H^{2} \frac{d B}{d z} \frac{d \bar{B}}{d z}+E c \frac{d F}{d z} \frac{d \bar{F}}{d z}=G F .
$$

The boundary conditions on $\Delta$ are then given by

$$
\Delta=0 \text { on } z=1 \text { and } \Delta=\theta_{0} \text { on } z=-1
$$

Substituting $\mathrm{F}$ and $\mathrm{B}$ in (3.18) and solving the resulting differential equation with the boundary conditions $(3.19 \mathrm{ab}), \tilde{\theta}(z)$ can readily be determined. However, since the solution for $\tilde{\theta}(z)$ is quite complicated, we avoid writing the long expression for it.

4. THE VELOCITY FIELD, MAGNETIC FIELD AND THE ASSOCIATED BOUNDARY LAYERS.

In order to investigate the salient feature of the boundary layers, we shall consider the following special cases related to various magnitudes of the Ekman and Hartmann numbers.

Case (i): $E \ll 1$ and $H^{2} \gg 1$.

To determine the flow field, the magnetic field and the associated boundary layers on the plate $z=1$, it is convenient to write $1-z=\zeta$ in (3.11) - (3.12), and then take the limit of the resulting expressions as $\zeta \rightarrow 0 \nu \rightarrow 0$ such that $\frac{\zeta}{\sqrt{v}}$ is finite. This leads to the following results:

$u+i v=a_{1}^{\prime}+\left(a_{2}^{\prime}+\frac{a_{3}^{\prime}}{\lambda^{2}}\right) \exp (-\alpha \zeta)(\cos \beta \zeta-i \sin \beta \zeta)-\frac{a_{3}^{\prime}}{\lambda^{2}}(1-\zeta)+\frac{S^{2} H^{2}}{2 \lambda^{4}}(1-\zeta)^{2} G \operatorname{Pm}$,

$H^{2}\left(B_{x}+i B_{y}\right)=\frac{i S H^{2}}{3 E \lambda^{4}}(1-\zeta)^{3} G P m+\frac{(1-\zeta)^{2}}{2 \lambda^{2}}\left[G H^{2}+\frac{2 i S P m}{E}\left(\frac{2 i a_{1}}{E}+R\right)\right]+\frac{2 i a_{1}^{\prime}}{E}$

$-\left(\frac{1-\zeta}{\lambda+R}\right) S G b_{2}-\left[\frac{H^{2}}{\lambda}\left\{a_{2}^{\prime}+\frac{2 b_{1} b_{2} S}{\lambda^{3}}(1+\delta)\right\}+\frac{b_{1} b_{2} b_{5}(1+\delta)}{\lambda^{4}}-\frac{G}{\lambda^{2}}+\frac{H^{2}}{\lambda^{3}}\left(G+2 S G b_{2}\right)\right.$ 
$\left.+\frac{S P m}{\lambda^{3}}\left(\frac{21 a 10}{E}-R\right)+\frac{b_{2} b_{5} G}{\lambda^{3}}-a_{10} \cdot\right] \exp (-\alpha \zeta)(\cos \beta \zeta-1 \sin \beta \zeta)+\frac{G H^{2} \delta}{2 \lambda^{2}}-\frac{G H^{2}}{2 \lambda^{2}}(1+2 \delta)$

$+\frac{H^{2}}{2}\left(B_{x 1}+B_{x 2}\right)+S^{2} \delta(1+\delta)\left[\frac{G b_{2}\left(1+b_{5}\right)}{\lambda^{3}}-a_{10}{ }^{\prime}+\frac{P m}{\lambda^{3}}\left(\frac{21 a_{10}}{E}-R\right)\right]$

$+\frac{1}{E \lambda^{2}}(1+2 \delta)\left(\frac{2 i a_{10}}{E}-R\right)$,

where $\alpha$ and $\beta$ are the real and imaginary parts of $\lambda$, and

$a_{1}^{\prime}=-a_{10}^{\prime}+s\left[2 b_{2} a_{10}^{\prime}-a_{11}^{\prime}-\frac{G H^{2} P m}{2 \lambda^{4}}\right], a_{2}^{\prime}=a_{10}^{\prime}+s\left[\frac{a_{11}}{a_{10}^{\prime}}-2 b_{2} a_{10}^{\prime}\right]$,

$a_{3}^{\prime}=G+S \operatorname{Pm}\left(\frac{21 a_{10}}{E}-R\right)$ and $a_{10} \dot{0}^{\prime}=\frac{b_{1}}{\lambda^{2}(1+\delta)}$

The solution (4.1) represents the steady hydromagnetic boundary layer flow which consists of the Ekman-Hartmann layer on the boundary plate. The non-dimensional thickness of this layer is of the order $\alpha^{-1} \equiv\left[\mathrm{H}^{2}+\left(\mathrm{H}^{4}+\frac{2}{\mathrm{E}^{2}}\right)^{\frac{1}{2}}\right]^{-\frac{1}{2}}$ which is in agreement with the result of Debnath [9] and also with that of Nanda and Mohanty [3] for the case $\mathrm{E} \ll 1$ and $\mathrm{H}^{2}=0(1)$. In particular, the Ekman-Hartmann layer reduces to the Ekman layer of thickness $O\left(E^{\frac{1}{2}}\right)$ or the Hartmann layer of thickness $O\left(\frac{1}{\mathrm{H}}\right)$ according as $\mathrm{H}^{2} \ll$ or $>\frac{2}{\mathrm{E}}$.

Case (ii): $E \ll 1$ and $H^{2} \sim 0(1)$

In this case, the solutions are obtained from (3.11) - (3.12), and have the form

$\begin{aligned} u=a_{1 r}+a_{3 r} & \zeta-\frac{1}{8} S G P m H^{2} E^{2} \zeta^{2}+\left[\left(a_{2 r}+\frac{1}{2} E a_{31}\right) \cos \left\{\frac{\zeta}{\sqrt{E}}\left(1-\frac{E H^{2}}{4}\right)\right\}\right. \\ & \left.+\left(a_{2 i}-\frac{1}{2} E a_{3 r}\right) \sin \left\{\frac{\zeta}{\sqrt{E}}\left(1-\frac{E H^{2}}{4}\right)\right\}\right] \exp \left\{-\frac{\zeta}{\sqrt{E}}\left(1+\frac{H^{2} E}{4}\right)\right\},\end{aligned}$

$v=a_{1 i}+a_{3 i}+\left[\left(a_{2 i}-\frac{1}{2} E a_{3 r}\right) \cos \left\{\frac{\zeta}{\sqrt{E}}\left(1-\frac{H^{2} E}{4}\right)\right\}\right.$

$\left.-\left(a_{2 r}+\frac{1}{2} E a_{3 i}\right) \sin \left\{\frac{\zeta}{\sqrt{E}}\left(1-\frac{E H^{2}}{4}\right)\right\}\right] \exp \left\{-\frac{\zeta}{\sqrt{E}}\left(1+\frac{E H^{2}}{4}\right)\right\}$,

$\mathrm{H}^{2} \mathrm{~B}_{\mathrm{x}}=\mathrm{s}(1-\zeta)^{2} \mathrm{~d}_{7}+\mathrm{d}_{8}(1-\zeta)+\left[\mathrm{d}_{13} \sin \left\{\frac{\zeta}{\sqrt{\mathrm{E}}}\left(1-\frac{\mathrm{EH}^{2}}{4}\right)\right\}\right.$ 
174

D.R.V.P. RHO, D.V. KRISHNA, AND L. DEBNATH

$$
\begin{aligned}
& \left.-d_{12} \cos \left\{\frac{\zeta}{\sqrt{E}}\left(1-\frac{E^{2}}{4}\right)\right\}\right] \exp \left\{-\frac{\zeta}{\sqrt{E}}\left(1+\frac{E^{2}}{4}\right)\right\} \\
& +\frac{H^{2}}{2}\left(B_{x 1}+B_{x 2}\right)+\frac{S P m}{2}(1+\delta)\left(\frac{b_{1}}{1+\delta}-R\right) \\
& -\frac{1}{85} S E^{\frac{5}{2}} H^{2} G(1+\delta)\left(5 d_{14}+\frac{3}{E} d_{15}\right), \\
H^{2} B_{y}=-d_{9}(1 & -\zeta)^{3}+d_{10}(1-\zeta)^{2}+d_{11}(1-\zeta)+\left[d_{13} \cos \left\{\frac{\zeta}{\sqrt{E}}\left(1-\frac{E H^{2}}{4}\right)\right\}\right. \\
& \left.+d_{12} \sin \left\{\frac{\zeta}{\sqrt{E}}\left(1-\frac{E H^{2}}{4}\right)\right\}\right] \exp \left\{-\frac{\zeta}{\sqrt{E}}\left(1+\frac{H^{2} E}{4}\right)\right\} \\
& -\frac{\delta E G H^{2}}{4}+\frac{1}{4} G E H^{2}(1+2 \delta)+\frac{3}{85} S E E^{\frac{5}{2}} H^{2}(1+\delta)\left(d_{14}-\frac{d_{15}}{E}\right) \\
& -\frac{1}{2} S E H^{2} R(1+\delta)\left(\frac{b}{1+\delta}-R\right),
\end{aligned}
$$

where $a_{1 r}, a_{1 i}$, etc. denote the real and imaginary parts of $a_{1}$ respectively, and

$$
\begin{aligned}
d_{3}= & \frac{3 d_{12} E^{\frac{5}{2}}}{16(1+\delta)}\left(\frac{2}{E}+H^{2} P m\right), \quad d_{4}=\frac{3 d_{12} E^{\frac{5}{2}}}{16(1+\delta)}\left(H^{2} P m-\frac{2}{E}\right), \\
d_{5}= & \frac{2 E^{3}}{85(1+\delta)}\left(\frac{8}{E}+H^{2} P m\right), \quad d_{6}=\frac{4 E^{3}}{85(1+\delta)}\left(2 H^{2} P m-\frac{1}{E}\right), \\
d_{7}= & \frac{P m}{2 E}\left(R-\frac{2 a_{11}}{E}\right), \quad d_{8}=R+\frac{S G P m H^{2} E^{2}}{4}-\frac{2 a_{1 i}}{E}, \\
d_{9}= & \frac{G P m H^{2} E}{12}, \quad d_{10}=\frac{S P_{1 r} a_{1 r}}{E^{2}}-\frac{G H^{2} E}{4}, \quad d_{11}=\frac{2 a_{1 r}}{E}+\frac{S G E}{2}, \\
d_{12}= & \frac{1}{2} H^{2} E\left[a_{2 r}-a_{2 i}-S b_{1}\left(d_{5}+d_{6}\right)\right]+d_{3}+\frac{3 G}{2 E}(1+\delta) d_{5} \\
& -H^{2}\left[\frac{G E}{4}+S G d_{5}(1+\delta)+\frac{S P_{m}}{4}\left\{\frac{b_{1}}{1+\delta}-R\right\} E E^{\frac{3}{2}}\right], \\
d_{13}= & \frac{1}{2} H^{2} E\left[a_{2 i}-a_{2 r}+S b_{1}\left(d_{5}+d_{6}\right)\right]-d_{4}+\frac{1}{2} G E \\
& -H^{2}\left[\frac{3}{4} G E^{\frac{3}{2}}-S G d_{6}(1+\delta)+\frac{S P m E^{2}}{4}\left\{\frac{b_{1}}{1+\delta}-R\right\}\right]+\frac{3 G}{2 E}(1+\delta) d_{5}+\frac{b_{1} E}{2(1+\delta)},
\end{aligned}
$$




$$
d_{14}=H^{2} P m+\frac{6}{85} E^{\frac{5}{2}}, \quad \text { and } \quad d_{15}=2-\frac{3 H^{2} \mathrm{PmE}^{3}}{85} \text {. }
$$

It is noted that the exponential terms in the above solution decays very rapidly as $\zeta$ increases and exceeds the value $\sqrt{\mathrm{E}} /\left(1+\frac{\mathrm{EH}^{2}}{4}\right)$. In the 1 imit $\zeta \rightarrow \infty$, the flow field and the magnetic field assumes the form

$$
\begin{aligned}
& u \sim a_{1 r}+a_{3 r} \zeta-\frac{1}{8} S G P \zeta^{2} E^{2} \\
& v \sim a_{1 i}+a_{3 i} \zeta \\
& \mathrm{H}^{2} \mathrm{~B}_{\mathrm{x}} \sim \mathrm{Sd}_{7}(1-\zeta)^{2}+\mathrm{d}^{8}(1-\zeta)+\frac{\mathrm{H}^{2}}{2}\left(\mathrm{~B}_{\mathrm{x} 1}+\mathrm{B}_{\mathrm{x} 2}\right)+\frac{\mathrm{S} \mathrm{Pm}}{2}(1+\delta)\left(\frac{\mathrm{b}_{1}}{1+\delta}-\mathrm{R}\right) \\
& -\frac{S P m}{2}(1+2 \delta)\left(\frac{b_{1}}{1+\delta}-R\right)-\frac{S G_{H}^{2}}{\sqrt{E}} \delta(1+\delta)\left(5 d_{14}+\frac{3 d_{15}}{E}\right), \\
& \mathrm{H}^{2} \mathrm{~B}_{\mathrm{y}} \sim-\mathrm{d}_{3}(1-\zeta)^{3}+\mathrm{d}_{10}(1-\zeta)^{2}+\mathrm{d}_{11}(1-\zeta)-\frac{\mathrm{GH}^{2} \delta \mathrm{E}}{4}+\frac{1}{4} \mathrm{GH} \mathrm{H}^{2} \mathrm{E}(1+2 \delta) \\
& +\frac{3 S G H^{2}}{\sqrt{E}}(1+\delta)\left(d_{14}-\frac{d_{15}}{E}\right)-\frac{S H^{2} R}{\sqrt{E}} \delta(1+\delta)\left(\frac{b_{1}}{1+\delta}-R\right) .
\end{aligned}
$$

The velocity field (4.3) - (4.4) shows the existence of the modified Ekman layer of thickness of the order $\sqrt{\mathrm{E}}\left(1+\frac{\mathrm{EH}^{2}}{4}\right)^{-1} \cdot$ It follows from (4.7) - (4.10) that both components of the velocity field persist, and are functions of the vertical coordinate $z$. This means the violation of the Taylor-Proudman theorem valid in an inviscid rotating fluid flows. Thus the suction or injection at the porous plates prevents the flow to reduce to two-dimensional. However, in the non-porous case the limiting flow satisfies the conditions of the Taylor-Proudman theorem. Case (iii): $\mathrm{H}^{2} \gg 1$ and $\mathrm{E}^{-1} \sim 0(1)$.

In this case, the solutions are given by

$$
\begin{aligned}
u & =f_{1}+\left(f_{2}+\frac{f_{3}}{H^{2}}\right) \exp (-H \zeta)-\frac{f_{3}}{H^{2}}+\frac{S G P m}{2 \zeta^{2}} \\
V & =f_{4}+\left(f_{5}+\frac{f_{6}}{H^{2}}\right) \exp (-H \zeta)-\frac{f_{6}}{H^{2}}, \\
H^{2} B_{x} & =\frac{1}{2 H^{2}}\left(G H^{2}-\frac{4}{E^{2}} S \operatorname{Pm} f_{7}\right)(1-\zeta)^{2}+\left[R-\frac{2 f_{4}}{E}-\frac{S G(1+P m)}{H^{2}}\right](1-\zeta)
\end{aligned}
$$




$$
\begin{aligned}
& -\left[H\left(f_{2}+\frac{2 f_{7}}{H^{4}}\right)-\frac{2 f_{7}}{H^{3}}-\frac{G}{H^{2}}+2 S G(1+P m)\right. \\
& \left.-\frac{S P m R}{H}-\frac{2 G}{H^{2}}(1+P m)-\frac{b_{1}}{H^{2}}(1+\delta)\right] \exp (-H \zeta) \\
& -G \delta+\frac{H^{2}}{2}\left(B_{x 1}+B_{x 2}\right)+S^{2} \delta(1+\delta)\left[\frac{G}{H^{4}}(1+P m)\left(1-2 H^{2}\right)\right. \\
& \left.-\frac{b_{1}}{H^{2}}(1+\delta)-\frac{R P m}{H^{3}}\right]-\frac{2 b_{1} P m(1+2 \delta)}{H^{4}(1+\delta) E^{2}},
\end{aligned}
$$

and

$$
\begin{aligned}
\mathrm{H}^{2} \mathrm{~B}_{\mathrm{y}}= & \frac{\mathrm{SGPm}}{3 \mathrm{EH}^{2}}(1-\zeta)^{3}-\frac{2 \mathrm{SPm} \mathrm{f}_{4}}{\mathrm{H}^{2} \mathrm{E}^{2}}(1-\zeta)^{2}+\frac{2 \mathrm{f}_{1}}{\mathrm{E}}(1-\zeta) \\
& -\left[\mathrm{Hf}_{5}+\frac{\mathrm{f}_{7}}{\mathrm{EH}^{5}}+\frac{2 \mathrm{SPm} \mathrm{b}_{1}}{\mathrm{E}(1+\delta) \mathrm{H}^{3}}+\frac{\mathrm{G}(1+\mathrm{Pm})}{\mathrm{EH}^{4}}\right] \exp (-\mathrm{H} \zeta) \\
& +\mathrm{SH} H^{3} \delta(1+\delta)\left\{\frac{\mathrm{G}(1+\mathrm{Pm})}{\mathrm{EH}^{4}}+\frac{2 \mathrm{Pm} \mathrm{b}_{1}}{\mathrm{EH}^{5}(1+\delta)}-\frac{\mathrm{SRPm}(1+2 \delta)}{\mathrm{EH}^{2}}\right\}
\end{aligned}
$$

where

$$
\begin{aligned}
\mathrm{f}_{1}=\frac{\mathrm{b}_{1}}{\mathrm{H}^{2}(1+\delta)}+\mathrm{S}\left(\frac{2 \mathrm{f}_{7}}{\mathrm{H}^{3}}-\mathrm{f}_{8}-\frac{\mathrm{GPm}}{2 \mathrm{H}^{2}}\right), \mathrm{f}_{2}=-\frac{\mathrm{b}_{1}}{\mathrm{H}^{2}(1+\delta)}+\mathrm{S}\left(\mathrm{f}_{8}-\frac{2 \mathrm{f}_{7}}{\mathrm{H}^{3}}\right), \\
\mathrm{f}_{3}=\mathrm{G}-\mathrm{SRPm}, \mathrm{f}_{4}=-\mathrm{Sf}_{9}, \mathrm{f}_{5}=\mathrm{Sf}_{8}, \mathrm{f}_{6}=\frac{2 \mathrm{Sb}_{1} \mathrm{Pm}}{\mathrm{E}(1+\delta) \mathrm{H}^{2}}, \mathrm{f}_{7}=\frac{\mathrm{b}_{1}(1+\mathrm{Pm})}{1+\delta} \\
\mathrm{f}_{8}=3 \delta\left(2 \mathrm{f}_{7}+\mathrm{G}\right)-\frac{3 \mathrm{G}}{\mathrm{H}^{2}}(1+\delta)(1+\mathrm{Pm}), \\
\mathrm{f}_{9}=\frac{G \mathrm{Pm}}{\mathrm{HE}}(1+3 \delta)-3 \delta\left(\frac{\mathrm{f}_{7}}{\mathrm{EH}^{2}}-\mathrm{G}\right)-\frac{\mathrm{G}(1+\delta)}{\mathrm{E}}\left(\frac{G \mathrm{Pm}}{2 \mathrm{H}}-\frac{2 \mathrm{f}_{7}}{\mathrm{H}^{2}}\right) .
\end{aligned}
$$

The solutions (4.11) - (4.14) represent the steady hydromagnetic boundary layers flow which consists of the Hartmann Layer of thickness of the order $\mathrm{H}^{-1}$. It is noted that the results for this case are completely independent of rotation.

\section{THE NUMERICAL RESULTS AND DISCUSSION.}

In order to present numerical results and their discussion, it is necessary to assume certain fixed values for $B_{x 1} \pm B_{x 2}, R$ and $\delta$. With $B_{x 1}+B_{x 2}=3.0$, $\mathrm{B}_{\mathrm{x} 1}-\mathrm{B}_{\mathrm{x} 2}=0.5, \mathrm{R}=1$ and $\delta=0.1$, the velocity, the temperature and the shear stresses are discussed numerically. The pressure gradient is taken to be positive 
along the direction of $x$ increasing, and hence the flow in the opposite sense indicated by negative $u$ is the actual flow whereas a positive $u$ corresponds to a reversed velocity. The velocity profiles for $u$ and $v$, the secondary flow, are presented for different values of the Hartmann number $H$, and the Grashof number $G$ for fixed $K\left(=\frac{1}{\sqrt{B}}\right)$ and $S$ as well as for different values of the suction parameter $S$ fixing $H, G$ and $K$. Figure 1 shows that in the absence of free convection ( $G \equiv 0$ ) for fixed $\mathrm{K}$ and small $\mathrm{S}$, as $\mathrm{H}$ increases the velocity profiles for $\mathrm{u}$ become more and more asymmetric with the point of maximum velocity shifting towards the upper half of the region. When $G \neq 0$, even for moderate values of $G, u$ is found to change its direction as we move from the upper plate to the lower one. The maximum value of $u$ is attained in the vicinity of the upper plate, and for large values of $G$, there is a sharp increase in the magnitude of $u$ both at the upper and the lower plates irrespective of the magnitude of $\mathrm{H}$. The region of reversed velocity near the lower plate gradually grows with the increase in G. However, for a fixed G, as $H$ increases the velocity near the upper plate enormously increases while the velocity at the lower plate decreases in magnitude.

The secondary velocity normal to the direction of the pressure gradient, as shown in Figure 2, behaves in the same manner as the primary velocity except that the regions of adverse velocity zones grow in size almost comparable to each other with increase in $G$ for a fixed $H$. However, for a fixed $G$ the reversed secondary velocity zone near the lower plate shrinks with the increase in $\mathrm{H}$. It has been pointed out earlier that the presence of suction eliminated the possibility of flow being two-dimensional in the central core for large $K$. It is interesting to observe the behavior of $\mathrm{u}$ and $\mathrm{v}$ for increase in the suction parameter $\mathrm{S}$ for fixed $\mathrm{H}, \mathrm{G}$ and $\mathrm{K}$. From Figure 3 it follows that while the magnitude of $\mathrm{u}$ increases rapidly with increase in $S$ there is a steady fall of $v$, although the maximum velocity of either of them tilt towards the upper plate similar to the $\mathrm{H}$ increasing case. For fixed values of $H, G$ and $S$, both $u$ and $v$ decrease as $K$ increases and for large $K$, a reversed flow develops near the lower plate as shown in Figure 4 . 


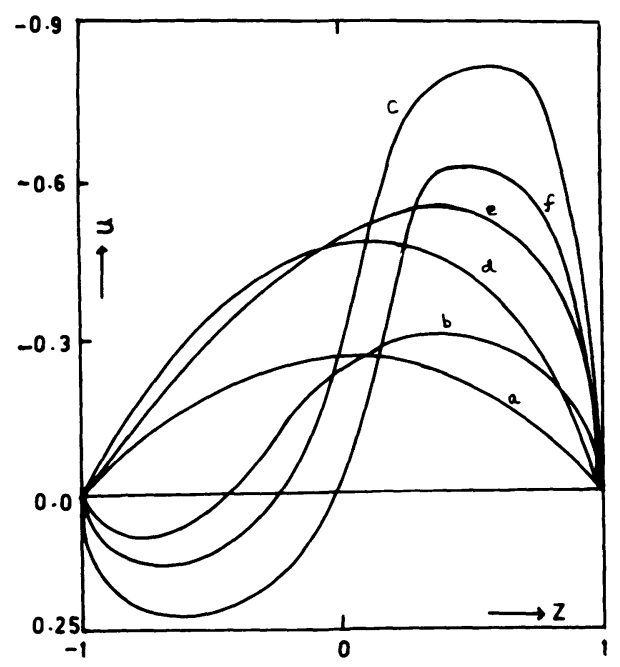

Fig. 1: Velocity profile for $u$ when $k=1, S=0.2$

$$
\begin{array}{rrrrrrr} 
& a & b & c & d & e & f \\
\text { H } & 0 & 0 & 0 & 2 & 2 & 2 \\
\text { G } & 0 & 4 & 10 & 0 & 4 & 10
\end{array}
$$

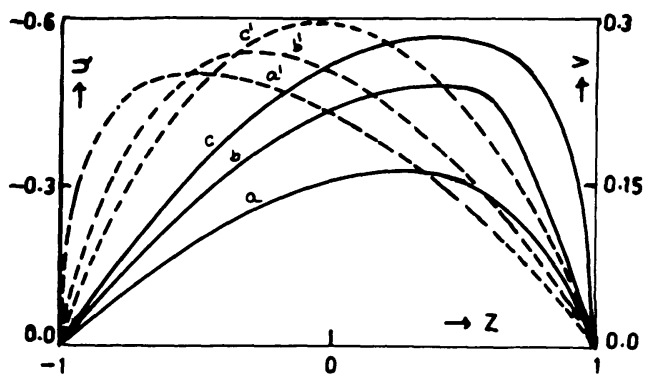

Fig. 3: $\mathrm{U}$ and $\mathrm{v}$ when $\mathrm{H}=1, \mathrm{G}=2, \mathrm{~K}=1$

$\begin{array}{lllllll}s & 0.0 & 0.2 & 0.4 & 0.0 & 0.2 & 0.4\end{array}$

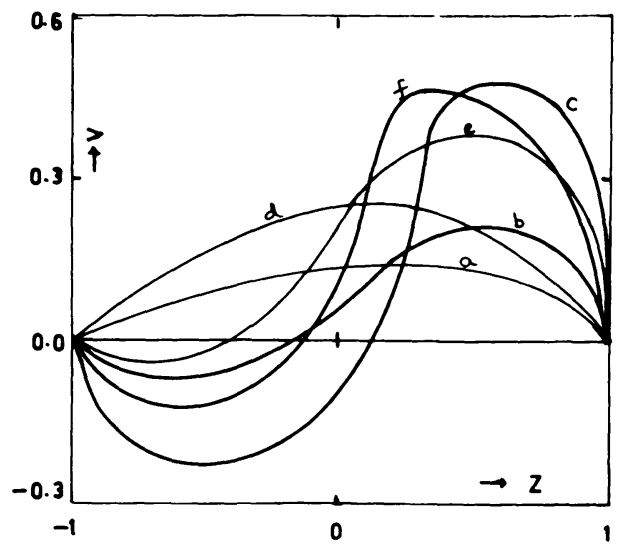

Fig. 2: Velocity profile for $v$ when $K=2, S=0.2$

$$
\begin{array}{rrrrrrr} 
& \text { a } & \text { b } & \text { c } & \text { d } & \text { e } & \text { f } \\
\text { H } & 0 & 0 & 0 & 2 & 2 & 2 \\
\text { G } & 0 & 4 & 10 & 0 & 4 & 10
\end{array}
$$

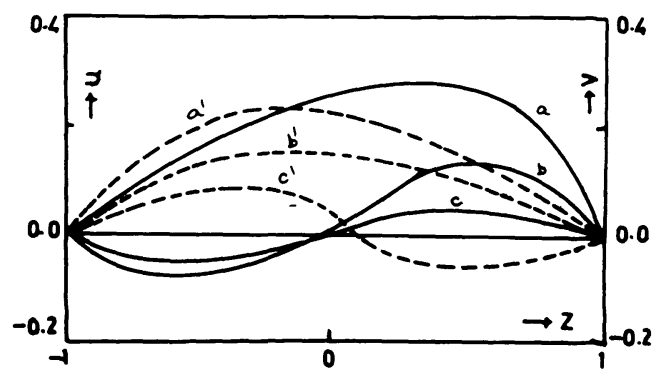

Fig. 4: $u$ and $v$ when $H=1, G=2, S=0.2$

$$
\begin{array}{ccccccc} 
& a & b & c & a^{\prime} & b^{\prime} & c^{\prime} \\
K & 1 & 2 & 3 & 1 & 2 & 3
\end{array}
$$


Figure 5 indicates the behavior of the dimensionless mass flow rates compared to $H$. The mass flow rate $Q_{x}$ along the $x$-axis rapidly increases with increase in $\mathrm{H}$ for a fixed $\mathrm{K}$ as well as increases with increase in $\mathrm{K}$ for a fixed $\mathrm{H}$. On the other hand, $Q_{y}$, for moderate values of $K$ retains the same nature but almost remains uniform for large $\mathrm{K}$.

The non-dimensional shear stresses $\tau_{x}$ and $\tau_{y}$ at the upper and lower plates are plotted in Figures 6-9 for various values of the governing flow parameters $S$, $H, G$ and $K$. It is to be noted that the reversed flow occurs whenever the shear stress on the upper and the lower plates are of the same sign. No such separation in the flow arises if the shear stress on the upper plate is opposite to that of the lower plate. Figure 6 shows that $\tau_{x}$ remains positive and decreases with increase in $\mathrm{H}$ for a fixed $G$, although increases for increase in $G$ for a fixed $H$. At the lower plate, it is negative and almost uniform for $G=0$. But as $G$ increases it remains negative for large values of $\mathrm{H}$ (say greater than 4) but becomes positive as $\mathrm{H}$ approches to zero. This shows that for $\mathrm{G} \neq 0$ and $\mathrm{H}=0$, reversed velocities appear in the vicinity of the lower plate. However, this region of reversed velocities which grows with increase in $G$ can be made to shrink by choosing sufficiently large $H$. The shear stress $\tau_{y}$ behaves similar to $\tau_{x}$ with occurence of the reversed velocities near the lower plate for smal1 H. Figures 8-9 indicate the behavior of $\tau_{x}$ and $\tau_{y}$ with respect to the variation of $S$ and $K$. We also observe that for $K=1$ no reversed flow appears with reference to either $u$ or $\mathrm{v}$ for all values of $\mathrm{S}$. When $\mathrm{K}$ is large for sufficiently small $\mathrm{S}$, we find its growth near the lower plate. For a fixed $S, \tau_{x}$ and $\tau_{y}$ at both the plates go on decreasing for an increase in $\mathrm{K}$. Also the shear stresses increase with $\mathrm{S}$ for a fixed $K$.

The non-dimensional temperature profiles are plotted in Figures 10-12 for different sets of parameters. In all these cases the profiles are almost similar except that the concavity increases with either increase in $\mathrm{S}$ or $\mathrm{G}$. For increase in either S,G and $H$ it can be noted that the profiles become more and more sharp contributing to the increase in the maximum temperature attained. 


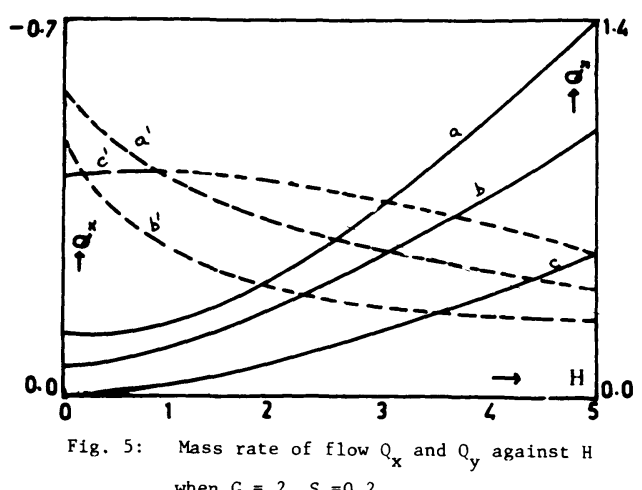

when $G=2, S=0.2$

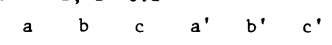

$\begin{array}{llllll}1 & 2 & 3 & 1 & 2 & 3\end{array}$

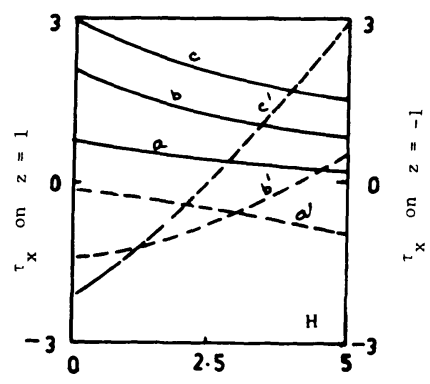

Fig. 6: $\tau_{x}$ against $H$ when $K=1, S=0.2$

$\begin{array}{lllllll}\text { G } & 0 & 4 & 10 & 0 & 4 & 10\end{array}$

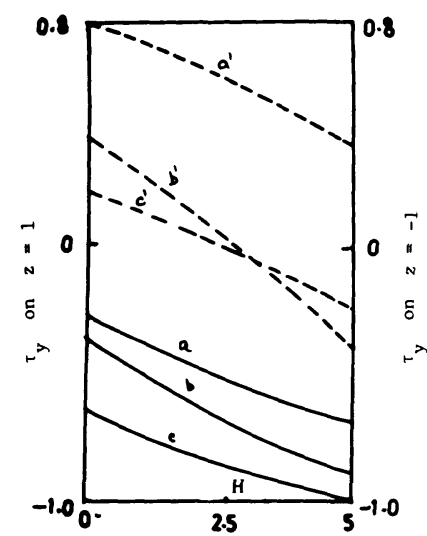

Fig. 7: $\tau_{\mathrm{y}}$ against $\mathrm{H}$ when $\mathrm{K}=2, \mathrm{~S}=0.2$ $\begin{array}{lllllll}\text { G } & 0 & 4 & 10 & 0 & 4 & 10\end{array}$

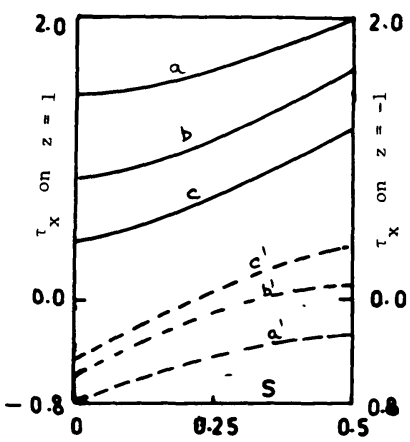

F1g. 8: $\tau_{x}$ against $S$ when $H=1, G=2$ a b c a $a^{\prime} \quad b^{\prime} \quad c^{\prime}$ $\begin{array}{lllllll}\mathrm{K} & 1 & 2 & 3 & 1 & 2 & 3\end{array}$ 


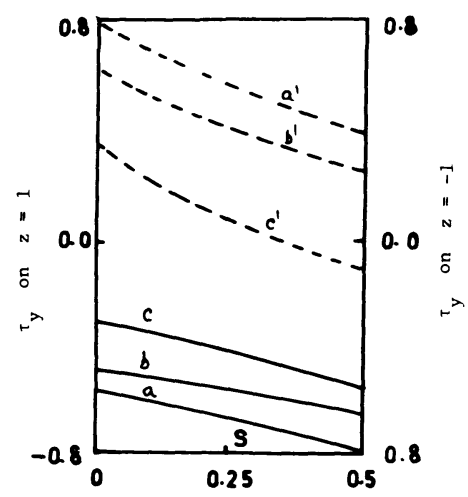

Fig. 9: $\tau^{\top} y$ against $S$ when $H=1, G=2$

- a b c $a^{\prime} b^{\prime} c^{\prime}$

$\begin{array}{lllllll}\mathrm{K} & 1 & 2 & 3 & 1 & 2 & 3\end{array}$

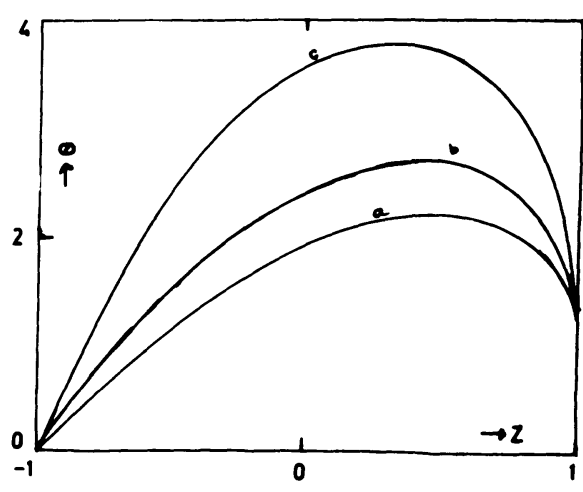

F1g. 10: Temperature distribution when $\mathrm{H}=1$,

$S=0.2, K=10$

$\mathrm{P}=1.5, \mathrm{Ec}=0.5, \mathrm{Pm}=1$

$$
\text { G } \quad 2 \quad 4 \quad 10
$$

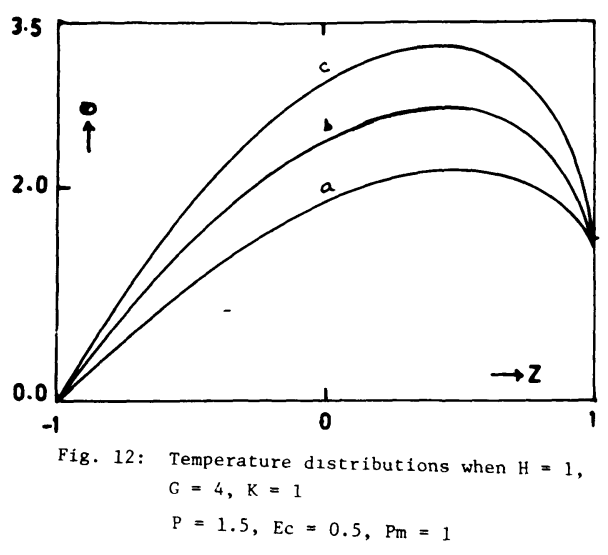

$\mathrm{P}=1.5, \mathrm{Ec}_{\mathrm{c}}=0.5, \mathrm{Pm}=1$

a b c

S $\quad 0.0 \quad 0.2 \quad 0.4$ 
ACKNOWLEDGEMENT. This work was partially supported by East Carolina University and the University of Oxford.

\section{REFERENCES}

1. Gupta, A.S. Combined free and forced convection effects on the magnetohydro- dynamic flow through a channe1, Z. Angew. Math. Phys. Vo1. 20 (1969) pp. 506-513.

2. Jana, R.N. Wall Conductance Effects on Convective Horizontal Channel Flow, Z.Angew. Math. and Phys. Vo1. 26 (1975) pp. 315-324.

3. Nanda, R.S. and Mohanty, H.K. Hydromagnetic Flow in a Rotating Channel, App1. Sci. Res. Vo1. 24 (1970) pp. 65-78.

4. Mishra, S.P. and Mudali, J.G. Combined free and forced convection effects on the magnetohydrodynamic flow through a porous channe1, Proc. Ind. Acad. Sci. Vol. 84 (1976) pp. 257-272.

5. Mohan, M. Combined effects of free and forced convection on magnetohydrodynamic flow in a rotating channel, Proc. Indian Acad. Sci. Vo1 $\underline{85}$ (1977) pp. 383-401.

6. Soundalgekar, V.M. On Heat Transfer in crossed fluids magnetohydrodynamic channel flow between conducting walls, Proc. Nat. Inst. Sci. India Vo1. A35 (1969) pp. 329-342.

7. Yen, J.T. Effect of Wall Conductance on Magnetohydrodynamic Heat Transfer in a Channe1, J. Heat Transfer, Transac. ASME. Vo1. $\underline{85 \mathrm{C}}$ (1963) pp. 371377.

8. Gill, W.M. and Casal, A.D. A Theoretical Investigation of Natural Convection Effects in Forced Horizontal Flows, Amer. Inst. Chem. Engg. Jour. Vo1. $\underline{8}$ (1962) pp. 513-520.

9. Debnath, L. On Ekman and Hartmann Boundary Layers in a Rotating Fluid, Acta. Mech. Vo1. 18 (1973) pp. 333-361.

*Present address: Mathematics Department

East Carolina University

Greenville, North Carolina 27834 U.S.A. 


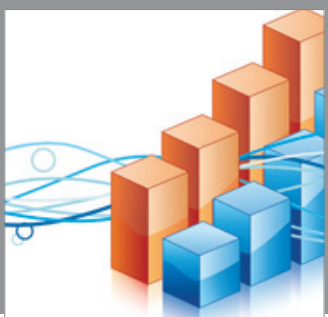

Advances in

Operations Research

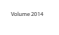

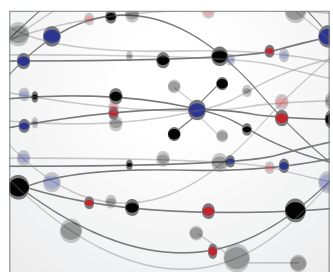

\section{The Scientific} World Journal
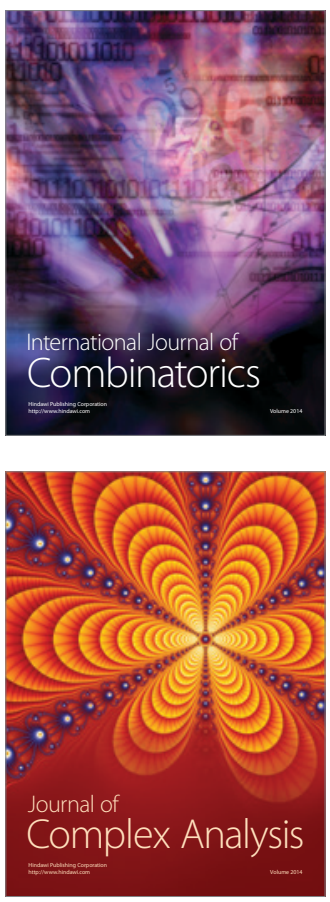

International Journal of

Mathematics and

Mathematical

Sciences
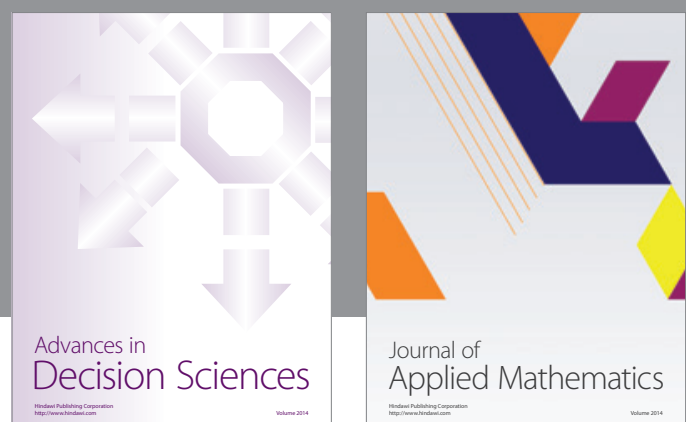

Journal of

Applied Mathematics
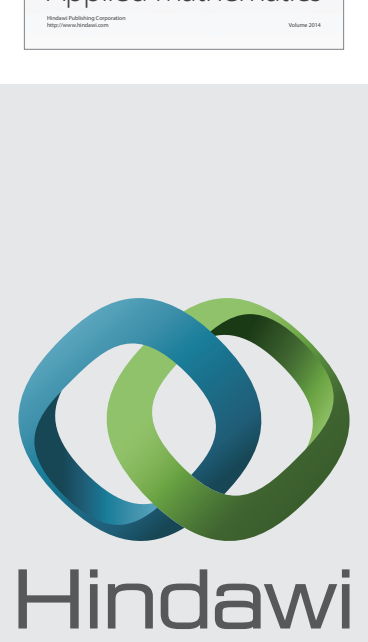

Submit your manuscripts at http://www.hindawi.com
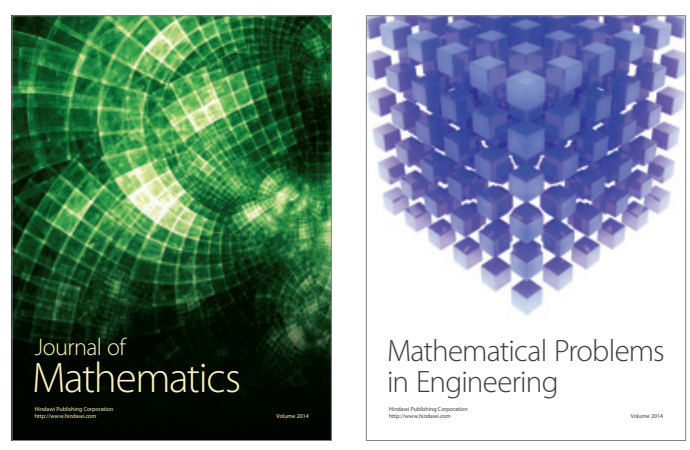

Mathematical Problems in Engineering
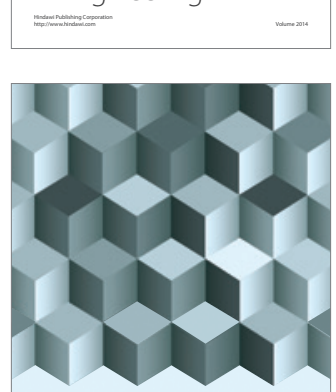

Journal of

Function Spaces
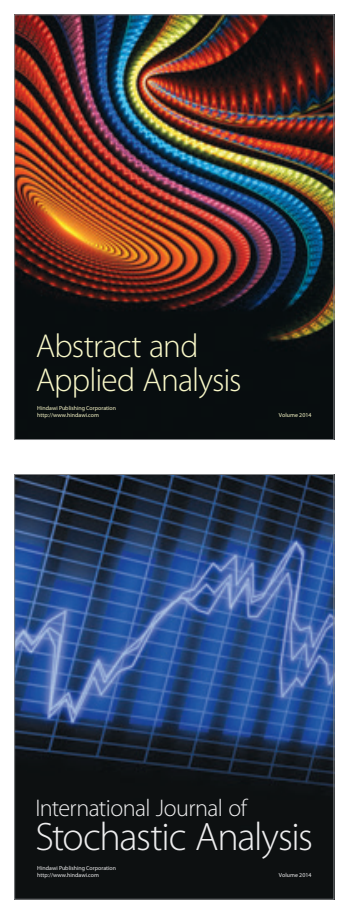

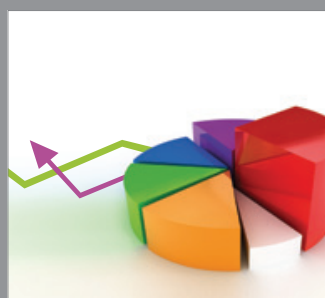

ournal of

Probability and Statistics

Promensencen
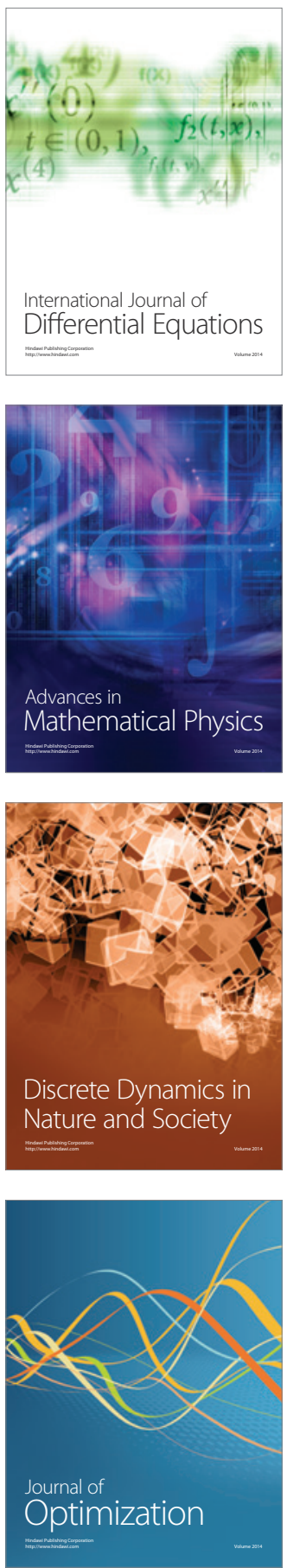